\title{
Successful behavior change in obesity interventions in adults: a systematic review of self-regulation mediators
}

Pedro J Teixeira ${ }^{1 *}$, Eliana V Carraça' ${ }^{1}$, Marta M Marques ${ }^{1}$, Harry Rutter ${ }^{2}$, Jean-Michel Oppert ${ }^{3,4}$, Ilse De Bourdeaudhuii ${ }^{5}$, Jeroen Lakerveld ${ }^{6}$ and Johannes Brug ${ }^{7}$

\begin{abstract}
Background: Relapse is high in lifestyle obesity interventions involving behavior and weight change. Identifying mediators of successful outcomes in these interventions is critical to improve effectiveness and to guide approaches to obesity treatment, including resource allocation. This article reviews the most consistent self-regulation mediators of medium- and long-term weight control, physical activity, and dietary intake in clinical and community behavior change interventions targeting overweight/obese adults.
\end{abstract}

Methods: A comprehensive search of peer-reviewed articles, published since 2000, was conducted on electronic databases (for example, MEDLINE) and journal reference lists. Experimental studies were eligible if they reported intervention effects on hypothesized mediators (self-regulatory and psychological mechanisms) and the association between these and the outcomes of interest (weight change, physical activity, and dietary intake). Quality and content of selected studies were analyzed and findings summarized. Studies with formal mediation analyses were reported separately.

Results: Thirty-five studies were included testing 42 putative mediators. Ten studies used formal mediation analyses. Twenty-eight studies were randomized controlled trials, mainly aiming at weight loss or maintenance $(n=21)$. Targeted participants were obese $(n=26)$ or overweight individuals, aged between 25 to 44 years $(n=23)$, and 13 studies targeted women only. In terms of study quality, 13 trials were rated as "strong", 15 as "moderate", and 7 studies as "weak". In addition, methodological quality of formal mediation analyses was "medium". Identified mediators for medium-/long-term weight control were higher levels of autonomous motivation, self-efficacy/barriers, self-regulation skills (such as self-monitoring), flexible eating restraint, and positive body image. For physical activity, significant putative mediators were high autonomous motivation, self-efficacy, and use of self-regulation skills. For dietary intake, the evidence was much less clear, and no consistent mediators were identified.

Conclusions: This is the first systematic review of mediational psychological mechanisms of successful outcomes in obesity-related lifestyle change interventions. Despite limited evidence, higher autonomous motivation, self-efficacy, and self-regulation skills emerged as the best predictors of beneficial weight and physical activity outcomes; for weight control, positive body image and flexible eating restraint may additionally improve outcomes. These variables represent possible targets for future lifestyle interventions in overweight/obese populations.

Keywords: Lifestyle interventions, Obesity, Self-regulation, Weight control, Physical activity, Dietary intake, Maintenance, Behavior change, Mediation analysis

\footnotetext{
* Correspondence: pteixeira@fmh.ulisboa.pt

${ }^{1}$ Interdisciplinary Centre for the Study of Human Performance (CIPER), Faculty

of Human Kinetics, University of Lisbon, Lisbon, Portugal

Full list of author information is available at the end of the article
}

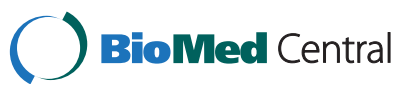

(c) 2015 Teixeira et al.; licensee BioMed Central. This is an Open Access article distributed under the terms of the Creative Commons Attribution License (http://creativecommons.org/licenses/by/4.0), which permits unrestricted use, distribution, and reproduction in any medium, provided the original work is properly credited. The Creative Commons Public Domain Dedication waiver (http://creativecommons.org/publicdomain/zero/1.0/) applies to the data made available in this article, unless otherwise stated. 


\section{Background}

Lifestyle treatment interventions for obesity typically target changes in diet and physical activity through strategies like setting adequate goals and enhancing patients' motivation, changing their beliefs and expectations, and providing guidance in the use of a variety of self-regulation skills (such as self-monitoring), all of which are thought to influence behavior change and maintenance [1-4]. A wide variety of health behavior change theories has been employed to provide conceptual organization of these determinants, including social cognitive theories such as the theory of planned behavior [5], theories of motivation such as self-determination theory [6], theories distinguishing between motivational and post-motivational or volitional phases [7] such as the health action process approach (HAPA) [8], and self-regulation models such as control theory [9]. Since all these theories address the regulation of a person's behavior in the service of some goal or desired outcome, through intrapersonal factors, in this paper we broadly refer to intervening variables in this process as self-regulation factors.

Behavior modification in general, and "comprehensive lifestyle interventions" in particular [10] are currently the first recommended step in obesity management. However, so far, randomized controlled trials evaluating the effectiveness of programs that target lifestyle behavior have shown mixed effects and, if effective, they have generally resulted in only small changes in target behaviors [11-15]. In addition, the evidence shows that relatively little if any weight loss accomplished in treatment programs is maintained over the long term [16]. Furthermore, few studies have analyzed why, or by which mechanisms, interventions are successful for some individuals and not for others. Clearly, there is a need for research that identifies causal predictors of long-term weight control, including successful weight loss and maintenance [17].

Despite the limited success of available interventions in reversing the current trends in obesity prevalence, approaches focusing on individual behavior change remain an important topic of interest in obesity research. Several reasons justify this assertion. First, these interventions typically focus on behaviors (for example, diet and physical activity), which have widespread consequences for health, with or without weight loss. Second, if and when individuals are able to successfully self-regulate their behaviors, these effects tend to be sustainable, which is essential for having a lasting impact on health; moreover, this successful self-regulation may also "transfer" to, and help change, other health behaviors [18]. Third, although some interventions targeting individuals may be ineffective on their own, they might be able to contribute to the effectiveness of strategies that integrate multiple levels (that is, strategies that include individuallevel and environmental-level approaches) [19]. Finally, the potential for dissemination of individual-level intervention approaches is considerable, given that a sizable number of overweight and obese persons will seek professional help at some point in their lives. Consequently, improving the efficacy of such interventions has substantial clinical as well as public health relevance.

One recent development in studies testing lifestyle interventions for obesity is their ability to identify the mechanisms or processes by which interventions induce meaningful and lasting change in their (most successful) participants. These mechanisms can generally be named predictors (or determinants) of success, and some studies have gone one step further to evaluate the extent to which they may be causal mediators of intervention effects. Testing of mediation, using appropriate methods, is a critical step in this field; it provides the strongest possible inference for the identification of elements in interventions which are causally "responsible" for achieving desired outcomes [20].

Success and failure in the self-regulation of health behaviors involve multiple psychological and behavioral aspects. The aim of this review was to identify and summarize psychological self-regulation mediators of successful weight change, or change in energy balance-related behavior (physical activity and diet), in clinical and community behavior change obesity interventions. Because eventual weight regain is frequent after behavior and/or weight change interventions, particular attention was given to studies reporting long-term outcomes, that is, one year or more after the beginning of the intervention.

\section{Methods}

This systematic review was conducted in accordance with the Preferred Reporting Items for Systematic Reviews and Meta-Analyses (PRISMA) statement [21].

\section{Eligibility criteria}

Studies were included in this review if they were intervention studies published since 2000 in the English language, used experimental designs, and referred to clinical or community behavior change interventions with overweight/obese adults ( $\geq 18$ years old) aiming to reduce overweight/obesity. This review was limited to "lifestyle interventions" defined as interventions that promote change in energy balance-related behaviors (such as diet and physical activity, as the outcomes) and self-regulatory factors (such as motivation and self-monitoring, as the potential mediators) relevant for overweight/obesity treatment, typically in settings involving personal contact between interventionists and participants. There were no restrictions with respect to the format and duration of the intervention. To be eligible, studies should also report outcomes assessed at least 6 months after the start of the intervention; include a quantitative assessment of change 
in weight/BMI, physical activity (for example, selfreported or accelerometer-derived minutes of moderate and vigorous physical activity, daily pedometer steps, attendance to PA sessions), or dietary intake (for example, energy intake, fat intake, fruit and vegetable intake) as well as a quantitative assessment of potential mediators of successful behavior change. We decided not to distinguish predictors of weight loss and predictors of weight loss maintenance, choosing instead to divide the studies according to the length of measurement periods (shorter versus longer than 12 months). While it is possible that predictors of those two processes differ, to appropriately evaluate predictors of weight loss maintenance, we would have to rely on studies of successful weight losers, and preferably including psychological measures before and after the maintenance period. Only one intervention study fit both criteria.

An a priori list of mediators was used for study inclusion/exclusion, based on previous work in this area (for example, [2,22]). Only mediators representing individuallevel self-regulatory processes were considered (that is, those related to skills, motivation, competence, coping mechanisms, beliefs, physical self-perceptions, and eating regulation factors such as disinhibition, restraint, and perceived hunger). Mediators associated with personality factors, social support, and health-related outcomes (such as psychological distress, quality of life, and well-being) were excluded. Finally, eligible studies were required to report the effect of the intervention on hypothesized mediator(s) and the association of the putative mediator with the outcomes of interest.

\section{Search strategy and study selection}

A comprehensive search of peer-reviewed articles published between January 2000 and February 2014 (including online ahead of print publication) was conducted in six electronic databases (Pubmed, MEDLINE, PsycINFO, the Cochrane Library, Web of Knowledge, and SPORTDiscus). The decision to restrict the selection to studies published since 2000 is based on the fact that recent development in studies testing the effectiveness of lifestyle interventions for obesity makes older studies less externally valid. For instance, in 1995, Friedman and Brownell [23] alerted for the need of a "third generation" of obesity treatment studies analyzing causal mechanisms between psychosocial variables and weight change. Despite this, one decade later, it has been observed that very few studies had investigated such mechanisms, and even fewer looked into long-term changes [2].

Searches included various combinations of four sets of terms: i) terms concerning the health condition or population of interest (overweight/obesity); ii) terms concerning the intervention(s)/exposure(s) evaluated (for example, behavior change/lifestyle obesity interventions); iii) terms respecting the outcomes of interest (weight change, physical activity, and dietary intake); iv) terms concerning the predictors/mediators of interest (psychological, selfregulation); and v) terms concerning the type of analyses of relevance (for example, mediation, correlates, predictors). (See Additional file 1 for a search example; complete search strategies can be obtained from the authors). Other sources included manual cross-referencing of bibliographies cited in previous reviews [2,22,24-26] and included studies, as well as manual searches of the content of key scientific journals (Obesity Reviews; International Journal of Obesity; Obesity (Silver Spring); International Journal of Behavioral Nutrition and Physical Activity; Journal of the American Dietetic Association; Psychology of Sports and Exercise; Health Psychology; Journal of Behavioral Medicine; Preventive Medicine).

Titles, abstracts, and references of potential articles were reviewed by two authors (EVC, MM) to identify studies that met the eligibility criteria. Duplicate entries were manually removed. Relevant articles were then retrieved for a full read. The same two authors reviewed the full text of potential studies, and decisions to include or exclude studies in the review were made by consensus.

\section{Data coding and extraction}

A data extraction form was developed, informed by the PRISMA statement for reporting systematic reviews [21] and the Cochrane Collaboration's tool for assessing risk of bias [27]. Data extraction included information about study details (authors, year, country of publication, affiliations, and funding), participants (characteristics, recruitment, setting, attrition, compliance, and blinding), study design and setting, outcomes of interest, mediators/ predictors (in/out list), intervention length and characteristics, psychosocial instruments, and statistical analysis, including mediation techniques (a complete coding form can be obtained from the authors). Authors of included studies were contacted when necessary to retrieve missing data in published reports.

Considering that the main focus of this review was the identification of mediators, data extraction was performed separately, starting with the studies formally testing mediation (see Additional file 2), followed by those that reported both the effect of the intervention on hypothesized mediators (path $a$ ) and the association of the putative mediator with the outcomes of interest (path $b$ ), but did not test mediation (see Additional file 3). Regarding mediation and specifically in studies with formal mediation tests, researchers could use Baron and Kenny's approach [28] and check whether the main effects were reduced in the presence of the mediator, or employ more sophisticated techniques to directly test the significance of the indirect effect through the mediator (for instance, by following MacKinnon's approach [29], and 
using Preacher and Hayes mediation procedures or structural equation modeling). Additional file 4 presents a detailed description of the mediation analyses procedures and estimates for each study. In the latter (that is, predictor studies), we generally looked at a) whether significant intervention-control differences existed for a given variable (or pre-post change in non-controlled designs); b) whether there was an association between these changes (in intervention group only) and changes in the outcome (weight/PA/diet) in this group. If both were present, results were deemed consistent with mediation.

\section{Quality assessment}

The quality of included studies was assessed using an adapted version of the Quality Assessment Tool for Quantitative Studies, developed by the Effective Public Health Practice Project [30], and recommended for use by the Cochrane Public Health Review Group [27]. The current adaptation was based on recommendations from several authors [31,32], and has been used in a previous systematic review conducted as part of the SPOTLIGHT project [33]. This tool was adapted to allow the evaluation of both experimental and observational studies and contains 19 items, guiding the assessment of eight key methodological domains - 1) study design, 2) blinding, 3) representativeness (selection bias), 4) representativeness (withdrawals/dropouts), 5) confounders, 6) data collection, 7) data analysis, and 8) reporting. Each domain is classified as Strong (low risk of bias/high methodological quality), Moderate, or Low (high risk of bias/ low methodological quality) methodological quality. A global rating is determined based on the scores of each component (see Additional file 5 for a full description of the Assessment Tool components and scoring system). Two researchers independently rated each of the eight domains and overall quality (EVC, MM). Discrepancies were resolved by consensus.

For studies employing formal tests of mediation, assessment of methodological quality was complemented with a checklist tool developed specifically for mediation analysis by Lubans, Foster, and Biddle [34], and subsequently adapted by Rhodes and Pfaeffli [35]. This tool includes 11 questions answered with a yes (1) or no (0) format, whose scores are added to generate a global score. High quality is represented by scores between 9 and 11, moderate quality ranges between 5 and 8 , and low quality is considered when scores are below 5 (see Additional file 5 for a full description of the Checklist components and scoring system). Methodological quality of the mediation analyses was also rated by two authors (EVC, MM), with conflicting judgments discussed to reach agreement. Inter-rater agreement was good (Cohen's kappa $=0.78$ ).

\section{Data synthesis}

This review analyzed psychological and self-regulation mediators and predictors of change in body weight or BMI (primary outcome), physical activity, and dietary intake, separately (Note: we will use the term predictors when studies did not test for formal mediation, and mediators when they did). Intervention effects on the outcomes of interest were included in Additional files 2 and 3 . Results were divided according to the length of assessment of the outcomes, into short-term ( $<12$ months from the start of the intervention) and long-term $(\geq 12$ months), so that those variables mediating/predicting more sustainable outcomes (the main focus of this review) could be more easily identified. Twelve months has been indicated by an expert panel on obesity as an appropriate threshold between weight loss and the maintenance of the weight lost [10]. In the synthesis of data derived from studies formally testing mediation, only controlled trials were included, to further strengthen inference regarding intervention effects on mediators and outcomes. In the case of prediction studies (not formally testing mediation), we included both controlled and uncontrolled trials, to capitalize on the (relatively) larger number of studies available, which would otherwise be excluded using the more stringent criteria. Table 1 describes the 35 included studies. In Tables 2, 3, 4, 5, 6, and 7 , mediation-specific results are discriminated from the general results, provided that the main goal of this review was the identification of self-regulation mediators in behavior change obesity interventions. The overall results (considering multivariate, bivariate/correlational, and mediation analyses) are also presented in each table (Tables 2, 3, 4, 5, 6, and 7). A total of 42 mediators/predictors were identified across outcomes. To facilitate data interpretation, considering the very large number of individual variables, these were grouped together by similarity into categories. Categorization was done through the extraction of information from primary studies on the definition and operationalization of the constructs. The following 12 categories were formed: Self-regulatory skills use, Processes of change, Coping mechanisms, Self-efficacy/barriers, Autonomous motivation, Controlled motivation, Decisional balance, Outcomes expectations/beliefs, Body image/physical self-worth, Cognitive restraint, Eating disinhibition, Perceived hunger. Please refer to Additional file 6 for full details regarding the mediators/predictors identified per outcome.

Finally, Tables 2, 3, 4, 5, 6, and 7 show, separately for each mediator/predictor, the number of studies that have analyzed it, the number of times it was tested (some of them within the same study), and the number of times a significant effect was found. Results are presented for mediation-specific results and for the overall results. 
Table 1 Characteristics of intervention studies examining potential mediators/predictors of weight control, physical activity, and food intake ( $n=35$ )

\begin{tabular}{|c|c|c|c|}
\hline Characteristics & $\begin{array}{l}\text { Number of } \\
\text { studies }\end{array}$ & Characteristics & $\begin{array}{l}\text { Number of } \\
\text { studies }\end{array}$ \\
\hline $\begin{array}{l}\text { Study design } \\
(n=35)\end{array}$ & & $\begin{array}{l}\text { Intervention } \\
(n=35)\end{array}$ & \\
\hline Trial & & Aim & \\
\hline $\mathrm{NCT}$ & 6 & Weight loss & 15 \\
\hline $\mathrm{NRCT}$ & 1 & Weight maintenance & 6 \\
\hline $\mathrm{RCT}$ & 28 & Exercise adherence & 3 \\
\hline Arms & & Health promotion & 8 \\
\hline $1 \mathrm{arm}$ & 6 & Other & 2 \\
\hline 2 arms & 18 & Theoretical grounds ${ }^{b}$ & \\
\hline 3 arms & 8 & $\mathrm{SCT}$ & 23 \\
\hline 4 or + arms & 3 & TाM & 5 \\
\hline Sample size & & SDT & 3 \\
\hline$<100$ & 2 & Other & 8 \\
\hline 100-199 & 12 & N.R. & 4 \\
\hline $200-299$ & 11 & Intervention setting & \\
\hline \multirow[t]{2}{*}{$\geq 300$} & 10 & University & 15 \\
\hline & & Hospital/clinic & 3 \\
\hline $\begin{array}{l}\text { Participants } \\
(n=35)\end{array}$ & & $\begin{array}{l}\text { Exercise/fitness } \\
\text { club }\end{array}$ & 12 \\
\hline Gender & & Community & 3 \\
\hline Women only & 13 & Web & 1 \\
\hline Both genders & 22 & Other & 1 \\
\hline Mean age, years & & Intervention length & \\
\hline $25-44$ & 23 & $<6$ months & 6 \\
\hline $45-64$ & 11 & 6-12 months & 16 \\
\hline N.R. & 1 & 12-24 months & 12 \\
\hline$B M l$ & & $\geq 24$ months & 1 \\
\hline $25-29.9$ & 2 & Post-intervention follo & $o w-u p(n=11)$ \\
\hline $30-34.9$ & 14 & $<6$ months & 1 \\
\hline $35-39.9$ & 4 & 6-12 months & 5 \\
\hline$\geq 40$ & 8 & 12-24 months & 4 \\
\hline N.R. & 7 & $\geq 24$ months & 1 \\
\hline
\end{tabular}

Table 1 Characteristics of intervention studies examining potential mediators/predictors of weight control, physical activity, and food intake $(\mathbf{n}=\mathbf{3 5})$ (Continued)

\begin{tabular}{|c|c|c|c|}
\hline Characteristics & $\begin{array}{l}\text { Number of } \\
\text { studies }\end{array}$ & Characteristics & $\begin{array}{l}\text { Number of } \\
\text { studies }\end{array}$ \\
\hline $\begin{array}{l}\text { Mediation analysis } \\
(n=10)\end{array}$ & & Outcomes & \\
\hline Type of test & & $\begin{array}{l}\text { Weight change } \\
(n=26)\end{array}$ & \\
\hline Regression-based & 2 & $\begin{array}{l}\text { Short-term } \\
\text { (<12 months) }\end{array}$ & 9 \\
\hline $\mathrm{P}$ and $\mathrm{H} /$ bootstrap & 4 & $\begin{array}{l}\text { Long-term } \\
\text { ( } \geq 12 \text { months) }\end{array}$ & 17 \\
\hline SEM/bootstrap & 4 & & \\
\hline \multicolumn{2}{|c|}{ Mediation approach (and criteria) ${ }^{a}$} & $\begin{array}{l}\text { Physical activity } \\
(n=19)\end{array}$ & \\
\hline Baron and Kenny & 3 & $\begin{array}{l}\text { Short-term } \\
\text { (<12 months) }\end{array}$ & 11 \\
\hline MacKinnon et al. & 7 & $\begin{array}{l}\text { Long-term } \\
\text { ( } \geq 12 \text { months) }\end{array}$ & 8 \\
\hline Shrout and Bolger & 2 & $\begin{array}{l}\text { Self-reported } \\
\text { (MVPA) }\end{array}$ & 15 \\
\hline \multirow[t]{2}{*}{ MacArthur } & 1 & Self-reported (LPA) & 2 \\
\hline & & $\begin{array}{l}\text { Self-reported } \\
\text { (Energy Exp.) }\end{array}$ & 1 \\
\hline \multicolumn{2}{|c|}{ Quality assessment score } & Objective ${ }^{c, d}$ & 4 \\
\hline \multicolumn{4}{|c|}{ Quality score (EPHPP tool; $n=35$ ) } \\
\hline Weak & 7 & $\begin{array}{l}\text { Food/energy intake } \\
(n=11)^{e}\end{array}$ & \\
\hline Moderate & 15 & $\begin{array}{l}\text { Short-term }(<12 \\
\text { months })\end{array}$ & 7 \\
\hline Strong & 13 & $\begin{array}{l}\text { Long-term ( } \geq 12 \\
\text { months) }\end{array}$ & 4 \\
\hline \multicolumn{2}{|c|}{ Quality score (Rhodes tool; $n=10$ ) } & Total energy intake & 3 \\
\hline Low & 0 & Fat/saturated fat & 6 \\
\hline Moderate & 10 & Fruit/vegetable & 7 \\
\hline High & 0 & Other (CH; protein) & 1 \\
\hline
\end{tabular}

Notes: NCT, non-controlled trial; NRCT, non-randomized controlled trial (for example, a study comparing two interventions, but without a real control group); RCT, randomized controlled trial (Note: two studies referred to post hoc analyses of existing RCTs, for outcomes that were not planned originally); N.R., not reported; $\mathrm{P}$ and $\mathrm{H}$, Preacher and Hayes mediation procedures; SEM, structural equation modeling; EPHPP, Effective Public Health Practice Project; $\mathrm{SCT}$, socio-cognitive theory; TTM, transtheoretical model; SDT, self-determination theory; MVPA, moderate and vigorous physical activity; LPA, lifestyle physical activity; Energy Exp., energy expenditure. ${ }^{a}$ Two studies used more than one mediation approach. ${ }^{b}$ Six studies used more than one theoretical framework. 'Objective assessments included accelerometry $(n=1)$, pedometry $(n=1)$, and actual attendance to a fitness club $(n=2) .{ }^{d}$ Two of the studies also assessed

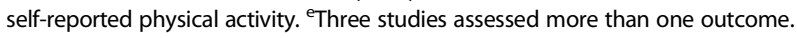

\section{Results}

Study selection

The literature search yielded a total of 1,394 potentially relevant records. Eight additional articles that were identified through manual searches and cross-referencing were added, bringing the total number of potential 
Table 2 Mediators/predictors of short-term weight control (<12 months)

\begin{tabular}{|c|c|c|c|c|c|c|}
\hline \multirow{2}{*}{$\begin{array}{l}\text { Putative mediators } \\
\text { (categories) }\end{array}$} & \multicolumn{3}{|c|}{ Formal mediation analyses } & \multicolumn{3}{|l|}{ All analyses } \\
\hline & Number of studies & Times tested & Effect, \% & Number of studies & Times tested & Effect, \% \\
\hline$\uparrow$ Self-regulation skill use & & & & 6 & 12 & $92^{\mathrm{a}}$ \\
\hline$\uparrow$ Self-efficacy/barriers & & & & 6 & 9 & $67^{* b}$ \\
\hline$\uparrow$ Body image/physical self-worth & & & & 2 & 6 & $67^{*}$ \\
\hline$\downarrow$ Eating disinhibition & & & & 1 & 4 & $75^{* c}$ \\
\hline$\uparrow$ Cognitive restraint & & & & 1 & 3 & $33^{* a}$ \\
\hline$\uparrow$ Processes of change & & & & 1 & 2 & $50^{*}$ \\
\hline$\uparrow$ Decisional balance (pros/cons) & & & & 1 & 1 & $100^{*}$ \\
\hline$\downarrow$ Perceived hunger & & & & 1 & 1 & $100^{* c}$ \\
\hline$\uparrow$ Positive outcome expectations & & & & 1 & 1 & 0 \\
\hline
\end{tabular}

Notes: Blank spaces correspond to variables that were not tested using formal mediation analyses. Times tested refers to the number of times a variable was analyzed; Effect, \% refers to the number of times an effect was found and is expressed as percentage. Since mediation analyses were not conducted, results are organized according to the number of times each variable was tested in the overall analyses, depicted in the fifth column, in descent order. ${ }^{*} \geq 50 \%$ of these

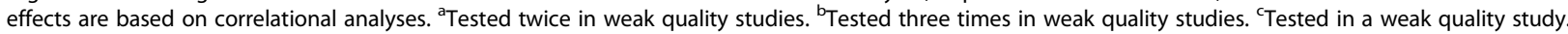
$[\uparrow]$ means that higher value of (variable) was associated with improved outcomes; $[\downarrow]$ means "lower value of (variable)" was associated with improved outcomes.

articles to 1,404. Of these, 770 abstracts were assessed for eligibility (634 duplicates removed). After the initial screening of titles and abstracts, 692 articles were excluded (Figure 1). Some articles were excluded for multiple reasons. Thirty-five articles describing 32 unique lifestyle interventions met the eligibility criteria and were therefore included [36-70]. Papers reporting on the same intervention are identified in Additional files 2 and 3.

\section{Study characteristics}

The characteristics of included studies are summarized in Table 1 (for further details, see Additional files 2 and 3). Most studies $(n=28)$ were randomized controlled trials, mainly aiming at weight loss or weight loss maintenance $(\mathrm{n}=21)$. Most interventions took place in university $(\mathrm{n}=$ $15)$ or fitness club settings $(n=12)$, and most lasted more than 6 months $(n=29)$. However, only 11 trials included a follow-up assessment period and, of these, more than half were shorter than 12 months. Most studies were based on, or at least informed by, one or more theories of behavior change; the most frequent being social cognitive theory $(\mathrm{n}=23)$, the transtheoretical model $(\mathrm{n}=5)$, and selfdetermination theory $(n=3)$. Eight interventions were grounded in other theories, including group dynamics theory, problem solving model, theory of planned behavior, health belief model, and self-regulation theory. Four studies did not report using any theoretical framework. Samples were mostly composed of obese individuals $(n=26)$, aged

Table 3 Mediators/predictors of medium/long-term weight control ( $\geq 12$ months)

\begin{tabular}{|c|c|c|c|c|c|c|}
\hline \multirow[t]{2}{*}{ Putative mediators (categories) } & \multicolumn{3}{|c|}{ Formal mediation analyses } & \multicolumn{3}{|l|}{ All analyses } \\
\hline & Number of studies & Times tested & Effect, $\%$ & Number of studies & Times tested & Effect, \% \\
\hline$\downarrow$ Controlled motivation for PA & 1 & 4 & 0 & 1 & 8 & 0 \\
\hline$\uparrow$ Self-regulation skill use & 3 & 3 & 75 & 6 & 6 & $83^{a}$ \\
\hline$\uparrow$ Body image/self-worth & 2 & 3 & 100 & 4 & 34 & $62^{*}$ \\
\hline$\uparrow$ Self-efficacy/barriers & 2 & 3 & 67 & 6 & 28 & $68^{*}$ \\
\hline$\uparrow$ Autonomous motivation for PA & 1 & 2 & 100 & 2 & 8 & $100^{*}$ \\
\hline$\uparrow$ Flexible restraint & 1 & 2 & 100 & 2 & 5 & 60 \\
\hline$\uparrow$ Positive outcome expectations/beliefs & 1 & 2 & 0 & 3 & 6 & 50 \\
\hline$\downarrow$ Eating disinhibition & 1 & 1 & 100 & 3 & 16 & $38^{*}$ \\
\hline$\uparrow$ Cognitive restraint (total) & & & & 4 & 8 & $50^{*}$ \\
\hline$\downarrow$ Perceived hunger & & & & 3 & 5 & $20^{*}$ \\
\hline$\uparrow$ Coping mechanisms & & & & 1 & 2 & 0 \\
\hline
\end{tabular}

Notes: Blank spaces correspond to variables that were not tested using formal mediation analyses. Times tested refers to the number of times a variable was analyzed; Effect, \% refers to the number of times an effect was found and is expressed as percentage. Results are organized according to the number of times each variable was tested in mediation analyses, depicted in the second column, in descent order. For variables not tested in mediation analyses, the fifth column (times tested in overall

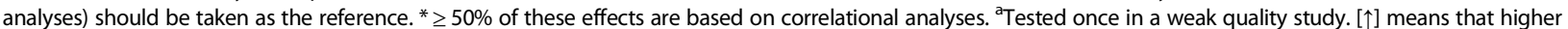
value of (variable) was associated with improved outcomes; [ $\downarrow]$ means "lower value of (variable)" was associated with improved outcomes. 
Table 4 Mediators/predictors of short-term physical activity ( $<12$ months)

\begin{tabular}{|c|c|c|c|c|c|c|}
\hline \multirow[t]{2}{*}{ Putative mediators (categories) } & \multicolumn{3}{|c|}{ Formal mediation analyses } & \multicolumn{3}{|l|}{ All analyses } \\
\hline & Number of studies & Times tested & Effect, \% & Number of studies & Times tested & Effect, \% \\
\hline$\uparrow$ Body image/physical self-worth & 1 & 2 & 50 & 3 & 6 & $67^{* a}$ \\
\hline$\uparrow$ Self-efficacy/barriers & 1 & 1 & 0 & 10 & 15 & $67^{*}$ \\
\hline$\uparrow$ Self-regulation skill usage & & & & 7 & 13 & $85^{*}$ \\
\hline$\uparrow$ Motivational readiness & & & & 1 & 2 & 50 \\
\hline$\uparrow$ Processes of change & & & & 1 & 2 & $50^{*}$ \\
\hline$\uparrow$ Decisional balance (pros/cons) & & & & 1 & 1 & $100^{*}$ \\
\hline$\uparrow$ Positive outcome expectations/beliefs & & & & 1 & 1 & 0 \\
\hline
\end{tabular}

Notes: Blank spaces correspond to variables that were not tested in formal mediation analyses. Times tested refers to the number of times a variable was analyzed; Effect, \% refers to the number of times an effect was found and is expressed as percentage. Results are organized according to the number of times each variable was tested in mediation analyses, depicted in the second column, in descent order. For variables not tested in mediation analyses, the fifth column (times tested in overall analyses) should be taken as the reference. ${ }^{*} \geq 50 \%$ of these effects are based on correlational analyses. ${ }^{\text {a }}$ Tested once in a weak quality study. [ $\uparrow$ ] means that higher value of (variable) was associated with improved outcomes.

between 25 to 44 years old $(\mathrm{n}=23)$, and 13 studies targeted women only.

Twenty-six studies evaluated mediators/predictors of weight change, of which 17 reported medium/long-term outcomes; 19 studies evaluated mediators/predictors of physical activity, with 8 of them reporting medium/longterm outcomes; finally, 11 studies investigated dietary intake as the outcome measure, 4 of them in the medium/ long-term. Weight-related measurements were performed with calibrated digital scales, and weight changes were expressed in weight change percent from baseline $(n=9)$, in kilograms $(n=10)$, as residualized scores regressed on the baseline scores $(n=6)$, or as BMI changes $(n=3)$. Regarding physical activity, objective measures were employed in 4 studies (for example, accelerometry, pedometry) and self-reported instruments in 17 studies; of these, 6 used the Seven-Day Physical Activity Recall [71] and 6 studies used the Godin Leisure-Time Exercise Questionnaire [72]. Dietary and caloric intake, indirectly assessed through the number of servings, was collected with the Food Intake Questionnaire in most studies $(\mathrm{n}=$ 5), followed by the Three-Day Food Records in most of the studies $(\mathrm{n}=3)$, and the Block Food-Frequency Questionnaire $(n=1)$.

\section{Quality assessment}

The overall results of the quality assessment can be found in Table 1 and the total quality score for each study in Additional files 2 and 3 (for a detailed classification of each item and study see Additional file 7). Regarding the overall methodological quality of the studies, 13 studies were rated as "strong", 15 were classified as "moderate", and 7 were rated as "weak". All included studies scored strong on the Study design, as they were experimental. Thirteen studies were rated as weak regarding Blinding of participants (during recruitment) and outcome assessors, 13 were rated as moderate, 8 as strong, and 1 did not receive a rating, as it was a non-

Table 5 Mediators/predictors of medium/long-term physical activity ( $\geq 12$ months)

\begin{tabular}{|c|c|c|c|c|c|c|}
\hline \multirow[t]{2}{*}{ Putative mediators (categories) } & \multicolumn{3}{|c|}{ Formal mediation analyses } & \multicolumn{3}{|l|}{ All analyses } \\
\hline & Number of studies & Times tested & Effect, \% & Number of studies & Times tested & Effect, \% \\
\hline$\downarrow$ Controlled motivation for PA & 2 & 8 & 0 & 2 & 12 & $25^{*}$ \\
\hline$\uparrow$ Autonomous motivation for PA & 2 & 6 & 83 & 2 & 14 & $93^{*}$ \\
\hline$\uparrow$ Self-efficacy/barriers & 4 & 6 & $67^{\mathrm{a}}$ & 6 & 12 & $75^{\mathrm{b}}$ \\
\hline$\uparrow$ Positive outcome expectations/beliefs & 2 & 3 & 0 & 3 & 3 & 0 \\
\hline$\uparrow$ Self-regulation skill usage & 2 & 2 & $50^{\mathrm{a}}$ & 3 & 3 & $67^{b}$ \\
\hline$\uparrow$ Coping mechanisms & 1 & 2 & 0 & 1 & 2 & 0 \\
\hline$\uparrow$ Decisional balance (pros/cons) & & & & 1 & 2 & 0 \\
\hline$\uparrow$ Cognitive restraint & & & & 1 & 3 & 0 \\
\hline$\uparrow$ Processes of change & & & & 1 & 2 & 0 \\
\hline
\end{tabular}

Notes: Blank spaces correspond to variables that were not tested in formal mediation analyses. Times tested refers to the number of times a variable was analyzed; Effect, \% refers to the number of times an effect was found and is expressed as percentage. Results are organized according to the number of times each variable was tested in mediation analyses, depicted in the second column, in descent order. For variables not tested in mediation analyses, the fifth column (times tested in overall analyses) should be taken as the reference. ${ }^{*} \geq 50 \%$ of these effects are based on correlational analyses. ${ }^{a}$ Tested once in a weak quality study. ${ }^{\mathrm{b}}$ Tested twice in weak quality studies. [ $\uparrow]$ means that higher value of (variable) was associated with improved outcomes; [ $\downarrow]$ means "lower value of (variable)" was associated with improved outcomes. 
Table 6 Mediators/predictors of short-term dietary intake ( $<12$ months)

\begin{tabular}{|c|c|c|c|c|c|c|}
\hline \multirow{2}{*}{$\begin{array}{l}\text { Putative mediators } \\
\text { (categories) }\end{array}$} & \multicolumn{3}{|c|}{ Formal mediation analyses } & \multicolumn{3}{|l|}{ All analyses } \\
\hline & Number of studies & Times tested & Effect, \% & Number of studies & Times tested & Effect, \% \\
\hline$\uparrow$ Self-efficacy/barriers & & & & 6 & 12 & $75^{*}$ \\
\hline$\uparrow$ Self-regulation skill usage & & & & 5 & 12 & $75^{*}$ \\
\hline$\uparrow$ Motivational readiness & & & & 1 & 4 & 0 \\
\hline
\end{tabular}

Notes: Blank spaces correspond to variables that were not tested in formal mediation analyses. Times tested refers to the number of times a variable was analyzed; Effect, \% refers to the number of times an effect was found and is expressed as percentage. Since mediation analyses were not conducted, results are organized according to the number of times each variable was tested in the overall analyses, depicted in the fifth column, in descent order. ${ }^{*} \geq 50 \%$ of these effects are based on correlational analyses. [ $\uparrow$ ] means that higher value of (variable) was associated with improved outcomes.

randomized trial. All studies except two (one scored weak and the other scored strong) scored moderate regarding Representativeness (selection bias). Regarding reporting of Withdrawals and dropouts, 5 studies were rated as weak, 16 as moderate, and 14 as strong. Four studies scored weak in the adjustment of analysis for Confounders, 10 scored moderate, and 21 strong. In terms of Data collection tools, 4 studies were rated as weak as they did not provide information on the validity or reliability of the measures used, 11 were classified as moderate, and 17 as strong. Three studies were not rated as they used a larger dataset for which information on psychometric properties of the measures is already provided. All studies scored strong in the use of Appropriate statistical analyses. In terms of Reporting, 30 studies were rated as strong, and 5 studies as moderate.

In addition, studies including formal tests of mediation $(n=10)$ were classified as of moderate $(n=10)$ quality on the mediation analysis checklist. None of the studies reported conducting pilot studies to test mediation, and in all except two studies, there was no specific information regarding the power of the analysis to detect mediation. In only six studies were the outcomes controlled for baseline values.

Mediators/predictors tested in studies with weak methodological quality are identified in Tables 2, 3, 4, 5, 6 , and 7. Overall, there appeared to be no association between the methodological quality of the studies and the results of the mediation analyses. Only 2 out of the 7 studies with a global weak score reported significant results for all mediator/predictors.

\section{Mediators/predictors of weight control}

Of the total number of studies investigating mediators/ predictors of weight control $(\mathrm{n}=26), 9$ looked into shortterm outcomes (<12 months) [47-49,51,52,54,57,62,70]. Twenty-one variables, grouped into nine categories, were tested as mediators/predictors of short-term weight control (Table 2). None of the studies performed formal tests of mediation. In the overall analyses (in this case, all were multivariate), self-regulation skill use emerged as the most consistent predictor of short-term weight control (consistent with mediation in $92 \%$ of the times it was tested [12 times in 6 studies]). Other variables that appear promising as mediators of short-term weight control were higher self-efficacy (and/or lower perceived barriers) and more positive body image, both consistent with mediation in $67 \%$ of the times they were tested (a total of 9 and 6 times, respectively). In the case of self-efficacy, 2 (out of 6) studies presented with low methodological quality. Although lower eating disinhibition also appears to find empirical support in non-formal mediation analyses, these results come from a single, weak quality study, and are

Table 7 Mediators/predictors of medium/long-term dietary intake ( $\geq 12$ months)

\begin{tabular}{|c|c|c|c|c|c|c|}
\hline \multirow[t]{2}{*}{ Putative mediators (categories) } & \multicolumn{3}{|c|}{ Formal mediation analyses } & \multicolumn{3}{|l|}{ All analyses } \\
\hline & Number of studies & Times tested & Effect, \% & Number of studies & Times tested & Effect, \% \\
\hline$\uparrow$ Self-efficacy/barriers & 1 & 2 & 50 & 3 & 8 & 25 \\
\hline$\uparrow$ Coping mechanisms & 1 & 2 & 0 & 2 & 4 & 0 \\
\hline$\uparrow$ Positive outcome expectations/beliefs & 1 & 1 & 0 & 2 & 2 & 0 \\
\hline$\uparrow$ Processes of change & & & & 1 & 8 & 0 \\
\hline$\uparrow$ Cognitive restraint & & & & 1 & 6 & 0 \\
\hline$\uparrow$ Decisional balance (pros/cons) & & & & 1 & 4 & 0 \\
\hline$\uparrow$ Self-regulation skill usage & & & & 2 & 2 & $100^{\mathrm{a}}$ \\
\hline
\end{tabular}

Notes: Blank spaces correspond to variables that were not tested in formal mediation analyses. Times tested refers to the number of times a variable was analyzed; Effect, \% refers to the number of times an effect was found and is expressed as percentage. Results are organized according to the number of times each variable was tested in mediation analyses, depicted in the second column, in descent order. For variables not tested in mediation analyses, the fifth column (times tested in overall analyses) should be taken as the reference. ${ }^{a}$ Tested twice in weak quality studies. [ $\uparrow$ ] means that higher value of (variable) was associated with improved outcomes. 


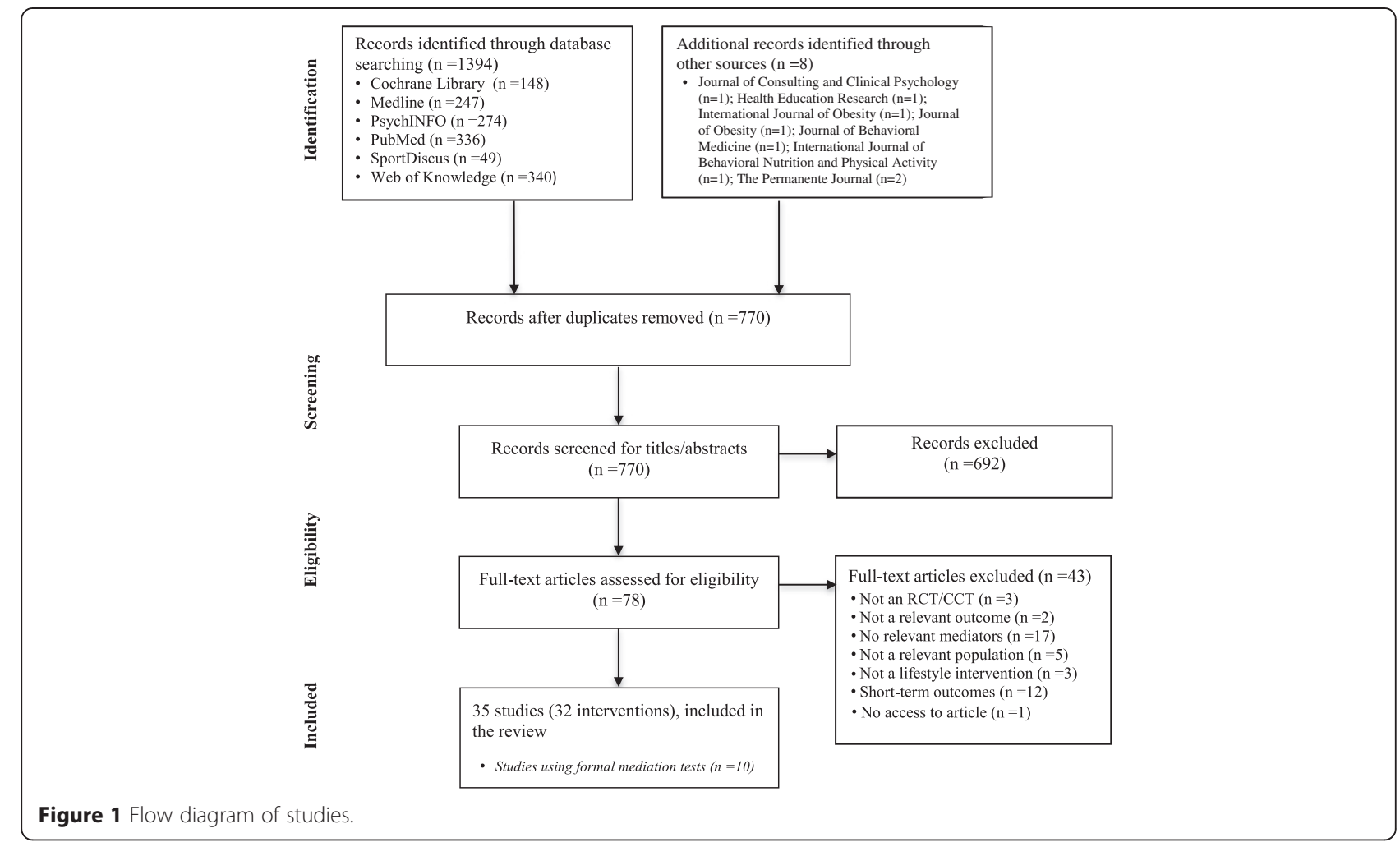

correlational in nature. There were no other consistent mediators/predictors of short-term weight control.

Seventeen studies investigated potential mediators/predictors of long-term ( $\geq 12$ months) weight outcomes, the main focus of the review [36,39,40,43-45,55,56,58,59,63-66]. Of these, six were RCTs that included formal tests of mediation $[36,39,40,43-45]$. Thirty variables, grouped in 12 categories, were tested as potential mediators/predictors (Table 3). The variables with stronger empirical support in formal mediation studies were body image, which was significant in all the times it was tested (3 times), and self-regulation skills, which was identified as a mediator in $67 \%$ of the times it was tested ( 2 times out of 3 studies). Self-efficacy was a significant mediator in 2 of the 3 times it was tested. For autonomous motivation and flexible eating restraint, results appear promising but derive from a single study in each case. Results observed in non-mediation analyses were consistent with the most stringent analyses, especially those concerning self-regulation skill use, autonomous motivation, and self-efficacy. For self-regulation skill use, significant effects were found in $83 \%$ of the 6 times it was tested, and every time in multivariate analyses. For autonomous motivation, results were consistent with mediation in all cases, but they originate from only two studies. On the other hand, empirical support from non-mediation analyses for other variables like body image and self- efficacy appears comparatively weaker and correlationbased; yet, the number of times each of these variables was tested is substantially higher (34 and 28 times, respectively). Eating disinhibition, which appeared to be an additional predictor in the short-term, does not seem to be consistent in the long-term provided that it was significant only in $38 \%$ of the 16 times it was tested. There were no other consistent mediators/predictors of long-term weight control.

\section{Mediators/predictors of physical activity}

Of the total number of studies investigating mediators/ predictors of physical activity $(n=19), 11$ looked into short-term outcomes (less than 6 months beyond the start of the intervention) [37,46,51-53,60,61,67-70]. Of these, only one formally tested mediation [37]. Fourteen variables, grouped in seven categories, were tested as mediators/predictors of short-term weight control (Table 4). Regarding mediation-specific results, body image emerged as a significant mediator only in one of the two times it was tested. In non-mediation studies, stronger empirical support was found for self-regulation skill use, which was significant in 11 of the 13 times it was tested (corresponding to 7 different studies). Body image and self-efficacy appear to be promising as mediators of short-term physical activity, showing significant results in 4 (out of 6) and 10 (out of 15) times they were tested, respectively. No other 
consistent mediators/predictors of short-term physical activity were identified.

Eight studies analyzed mediators/predictors of long-term physical activity $[36,38,41-43,50,64,65]$, of which five used formal tests of mediation [36,38,41-43]. Twenty-three variables, grouped in nine categories, were tested as predictors (Table 5). The main predictors of long-term physical activity were autonomous motivation and self-efficacy, considering both mediation-specific analyses and the overall analyses. For autonomous motivation, results from two studies showed that mediation analyses were significant in $83 \%$ of the times and overall analyses showed consistency with mediation in $93 \%$ of the times (out of 14). For self-efficacy, results originated from 6 different studies. Mediation analyses were significant in $67 \%$ of the times self-efficacy was tested (6 times); and in the overall analyses, results were consistent with mediation in $75 \%$ of the times (out of 12). Controlled motivation was also consistently unrelated with physical activity outcomes, independent of the type of analyses performed. Finally, self-regulation skill use appears to mediate long-term physical activity in one out of two (formal mediation) and two out of the three (all analyses) times tested, but these results derive from two studies with low methodological quality.

\section{Mediators/predictors of dietary intake}

Of the total number of studies $(\mathrm{n}=11)$ investigating mediators/predictors of dietary intake, seven looked into short-term outcomes [46,53,61,67-70] and four into long-term outcomes $[41,50,64,65]$. Only one study formally tested mediation [41]. Seven variables (grouped in three categories) were tested as mediators/predictors of short-term dietary intake, while 12 variables (grouped in seven categories) were tested in the long-term (Tables 6 and 7). Self-efficacy/barriers and self-regulation skill use appear promising as mediators of dietary intake in the short-term, both showing results consistent with mediation in $75 \%$ of the times they were tested (12 times out of 6 studies for self-efficacy, and 12 times out of 5 studies for self-regulation skills). No consistent mediators/ predictors were identified in the longer time frame. Yet, self-efficacy was consistently unrelated with long-term dietary intake, looking less promising as a mediator (results were consistent with mediation only in 2 of the 8 times it was tested).

\section{Discussion}

This review sought to identify the most consistent individual-level mediators of weight change, physical activity, and obesity-related dietary variables, in the context of lifestyle obesity interventions aimed at overweight and obese adults. These mediators or predictors of intervention effects were assessed by self-report, and are thought to represent psychological mechanisms or processes by which interventions affect body weight, through changes in energy-balance related behaviors. Note that this review did not focus on the efficacy of the interventions' main effects per se. However, mediation mechanisms can be evaluated even in the absence of main significant effects of interventions, particularly in controlled trials [20].

Special emphasis was given to variables tested as formal mediators of changes in the outcomes of interest, as this provides the best possible inferences regarding causal determinants of behavior change [73]; to the extent a consistent mediator is identified, it can more confidently be targeted in future interventions of comparable characteristics. Moreover, because it is unlikely that any single factor (self-regulatory or otherwise) by itself will explain a large share of variance of change in complex behaviors such as physical activity and diet (as a result of an intervention), the identification of groups of significant predictors, which can be then discussed in the context of current theories of behavior change, can additionally contribute to understanding the role of theory in health behavior change [74,75].

As in many systematic reviews of behavior change interventions, the diversity of studies available - reflected on a similarly diverse set of independent (and dependent) variables, study designs, measurement methods, populations represented, and so forth - is a substantial limitation. In the present review, the large number of predictors per study, combined with substantial heterogeneity in study length, type and format of interventions (for example, web-based, face to face, group-based), and assessments employed for each variable made the task at hand especially difficult. In this scenario, the fact that several variables were identified as predictors or, in some cases, actual mediators of intervention-related change in weight control and physical activity is encouraging. Specifically, the present review shows that positive changes in body image, in autonomous motivation for physical activity, in self-efficacy (and fewer perceived barriers), and in the use of self-regulation skills (such as self-monitoring) are promising aspects that may explain the variability of results in current lifestyle obesity treatment interventions. Increases in flexible restraint could also be in this group with respect to weight outcomes, but with lower inference. Therefore, these are currently the best evidence-based candidates to target in future individual-level, real-world interventions in this domain.

Some qualifications to the previous conclusions are of note. First, for short-term results, formal tests of mediation were only reported for one of the outcomes of interest (physical activity) and taking into account only two mediators (body image/self-worth and self-efficacy/ barriers). Second, there are currently too few studies using dietary variables as outcomes to allow us to draw 
meaningful conclusions, and only one study tested formal mediation for both time frames. Third, the external validity of some of the reported findings, such as regarding self-regulation skills and autonomous motivation, may be limited, because these findings were derived from few studies conducted by a small number of research groups, using similar study designs.

Body image appeared important as a mechanism leading to change in body weight in several studies. Body image is a multidimensional concept [76] that depicts attitudes, perceptions, and in some cases behaviors associated with mental representations of one's body (or some of its parts) [77,78]. Poor body image often reflects a high level of concern with body weight or shape, what is known as dysfunctional investment in body image, when body esteem occupies an excessive role as a determinant of overall self-esteem [79]. Previous reviews [2,22] have identified poor body image as a predictor of less success at body weight loss (or, conversely, better body image as a positive factor in obesity treatment interventions). Potential reasons for this association range from excessive psychological pressure leading to more rigid and inconsistent eating regulation [80-82] - poor body image being associated with a history of failed attempts and thus being a marker for other physiological, psychological, or socio-environmental risk factors for weight gain/regain $[83,84]$ - to motivational factors in which external pressures and goals predominate but tend not to produce behavior change in consistent or healthy ways (for example, wanting to be thin for reasons related to social comparison and perceived desirability) [85-87].

Autonomous motivation, a concept derived from selfdetermination theory (SDT, [88]), generally indicates the degree to which individuals self-endorse, feel that they have a choice about, and attribute deeply reflected value to a certain behavior. In contrast with the most common quantitative view of motivation (how much?), the level of autonomy represents a qualitative analysis of people's psychological energy to act, which is perceived as internal (reflecting a sense of "ownership" over the behavior). Autonomous motivation is often associated with goals such as pursuing positive personal challenges, attaining/preserving health and well-being, social affiliation, personal development, and self-expression [89]. Additionally, because self-determined, well-internalized behaviors are associated with the satisfaction of the needs for autonomy, competence, and relatedness - and with the feelings of internal coherence and well-being that are thought to emerge from those experiences - this provides an explanation for the behavior to be pursued consistently [89]. A recent meta-analysis [90] and other reviews provide empirical support for both the SDT motivation model and the association of autonomous motivation with health behavior change in different areas [91].
Self-efficacy and perceived barriers are common variables in several theoretical frameworks concerned with health behaviors [92,93]. Self-efficacy measures one's confidence to successfully implement a course of action by successfully organizing internal and external resources [94]. Although efficacy can be assessed towards other aspects of behavior regulation, it is commonly conceptualized and assessed as "barriers efficacy" or confidence to overcome internal or external obstacles that may stand in the way of one's actions. Indeed, the correlation between self-efficacy and perceived barriers is usually high [56] (which explains our decision to group these variables in the same category). Although conceptual differences exist, self-efficacy is often equated to the concept of perceived behavior control (from the theory of planned behavior) or perceived competence (as used in self-determination theory). In practical and simple terms, enhancing confidence and competence about a given health behavior appears to be helpful in overcoming barriers - namely in initial stages of adoption - and is often a first step to increase and improve motivation for change.

Flexible eating restraint involves regulating one's food intake so that no particular behavior is forbidden and thus subject to rigid control and scrutiny [95]. Flexible restraint is generally associated with less internal pressure to diet and a more gradual understanding of the diet's impact on energy balance. It stands in opposition to rigid restraint [96]. Although, in the past, cognitive restraint was measured as a unified concept, its separation into flexible and rigid dimensions is increasingly frequent in obesity studies and has proven useful in understanding diet and weight regulation, particularly in the long-term. For example, we found that flexible, but not rigid or total restraint, mediated 24-month weight loss in overweight women [39] and, in the present review, results for the total restraint scale and the flexible scale also differed, as in other studies [97]. More broadly, psychological flexibility appears to predict health and psychological well-being [97], is thought to reflect more committed, values-based goal pursuit $[98,99]$ and is considered a hallmark of self-determination [89], factors which may help explain successful health behavior self-regulation.

Finally, the use of certain self-regulation skills, for instance, monitoring weight, diet, and activity, as well as employing goal setting and planning techniques, was also identified as a relatively consistent predictor of successful outcomes, most especially in the shorter-term analyses. In brief, some of these skills may be important for people to ultimately act on their positive intentions. Sometimes associated with self-regulation theories ( $c f$. [100]) these variables are more skill-based (in some cases, they are discrete behaviors in themselves) and somewhat different than the previous set of predictors, more intrapsychic. Notably, recent behavior change models focusing 
on the "intention-behavior gap" (see, for example, $[7,101,102])$ make the distinction between motivational and implementation phases (sometimes referred to as "volitional" or "post-motivational"), with self-regulation skills reviewed in the present study falling in the latter phase [103]. Results from the present review suggest that some combination of motivational and implementation factors is important. Although this needs confirmation, there is some indication that the latter may be especially useful in early stages of behavioral adoption, whereas motivational factors may be operative along the entire continuum from adoption to maintenance, as highlighted recently in a separate study [104].

In looking at the collective findings from the present analysis, the temptation to interpret them in an integrative way is unavoidable. In principle, there should be "a logic" as to why this set of predictors emerged and not a different one, even considering the intrinsic limitations of the available data (see below). In this exercise, we are informed by our own research, for instance, linking improved eating regulation, including flexible eating, with improved body image [105] and with exercise autonomous motivation [18] and also by other studies. For example, recently, in a large dataset of women in New Zealand, autonomous motivation for eating was associated with less binge eating and slower speed of eating (and a much healthier diet), indicative of improved eating selfregulation [106]. The literature looking at relations between body image and eating behavior is also fertile in suggesting a close association between improved body image, improved eating regulation, and better weight control (see, for example, $[55,87])$. In this respect, an attempt was recently made to provide an integrative view of eating regulation and weight maintenance, which also includes an explanation for the etiology and role of body image concerns and disordered eating, while considering motivational and self-regulatory aspects [80]. It links goals (such as appearance versus health focus) and the predominant approach to eating regulation (such as rigid versus flexible restraint; focus on quality versus quantity) with the satisfaction of the needs for competence, autonomy, and relatedness, resulting in more or less adaptive diet and weight regulation (see Figures one and two in [80] for more details). The evidence from other recent systematic reviews and meta-analyses, showing that more autonomous forms of health behavior regulation, in physical activity [91], weight control [2], and in health more generally [90] are predictive of better adherence and improved outcomes, is also consistent with the relationships found in the present study.

While some limitations of the present work have been presented above, others need to be considered when interpreting the findings of this review. The large heterogeneity in the study-specific mediation methods and estimates reported in the primary studies prevented us from deriving a single comparable estimate for each variable and reporting on the pooled magnitude of mediation effects. This variability, as well as the limited number of studies for each mediator, did not recommend the use of meta-analytical techniques to pool data across studies. In this review, we used a narrative synthesis approach including vote counting of the number of significant mediation effects for a given variable in relation to all tests of mediation available for that variable. Although this method is not as robust as other quantitative approaches to synthesize data, it provides a reasonably good indication of whether that variable can be identified as a formal mediator (or a variable consistent with mediation) of each intervention, for each specific outcome. It should also be considered that in the primary studies included in this review, statistical significance of the mediation effects was typically the parameter used to infer that a given variable mediated the intervention effect.

Some studies were characterized by poor methodological quality, and none of the studies employing formal mediation analysis presented strong methodological quality. Nonetheless, for most mediators we did not find an association between the methodological quality of the studies and the direction/strength of the effects reported. As exceptions, we did find that in the analyses in which eating disinhibition had consistent significant effects, this was tested mostly in studies of poor quality. A similar result was found for self-regulation skills for the long-term effects in physical activity and dietary intake. Future reviews would benefit from sensitivity analyses. The diversity of outcome measures, especially for physical activity, is also a limitation, as different types of physical activity may be predicted by different factors. The fact that the coding of study characteristics was based on the description provided in the articles is also limiting, given that in many cases these descriptions did not provide enough information regarding mediation analysis, which measures were used, or the content of the interventions. Future studies should provide more detail on the content of the interventions and self-regulation factors addressed to facilitate data interpretation and inference. The choice of the year 2000 to start our search was largely arbitrary and could be seen as a limitation. Finally, the inclusion of noncontrolled trials in some of the analyses could be viewed as a limiting factor; on balance, we found this an acceptable compromise (for non-mediation studies only) against the prospect of altogether excluding several studies from this review.

These limitations notwithstanding, this study identified a small number of intervention-related aspects with supporting evidence for an important role played in the difficult path of successful weight control. Since all evidence was derived from intervention studies and independent 
variables were analyzed as to their mediating position in the behavioral causal chain (although with variable levels of inference), we believe this is a first step leading to their formal inclusion in recommendations for lifestyle programs aiming at weight control. In practical terms, this could mean that strategies or "behavior change techniques" [107] identified as the most effective to specifically change these variables (for example, self-efficacy [108] or autonomous motivation [109]) would be integrated into future interventions in a widespread fashion, and that health professionals would be appropriately trained on how to target them regularly in their practices. It could also mean that bedside instruments (such as brief questionnaires or interview items) would be made available for professionals to quickly assess their patients for these variables (for example, to assess their body image or level of self-regulation skill use $[1,110])$ and tailor interventions to the most relevant targets for each person. In the area of motivation enhancement, the techniques and instruments used in motivational interviewing [111,112] are a good example of such potential application in medicine and health care.

\section{Conclusion}

In conclusion, based on the scientific literature to date, autonomous motivation, self-efficacy, and self-regulation skill use emerge as the most promising individual-level mediators of positive weight outcomes and increased physical activity. For long-term weight control, promoting a positive body image and flexible eating control may also be important. These aspects represent potential entry points for future lifestyle obesity interventions in adults.

\section{Additional files}

Additional file 1: An Example of the Conducted Search (Medline). Additional file 2: Characteristics of Included Studies With Formal Mediation Analyses.

Additional file 3: Characteristics of Included Studies Without Formal Mediation Analyses.

Additional file 4: Indirect effects' estimates in studies with formal mediation analysis.

Additional file 5: EPHPP Quality Assessment Tool (adapted by the SPOTLIGHT Consortium).

Additional file 6: Complete results for weight change, physical activity and dietary behaviors.

Additional file 7: Consensus Ratings of Methodological Study Quality.

\section{Competing interests}

The authors declare that they have no competing interests.

\section{Authors' contributions}

PJT, JB, JL, JMO, HR, and IB conceived the study, and PJT and EVC developed a systematic review protocol. EVC and MM conducted the literature search and selected the studies based on the title and the abstract. EVC and MM extracted and coded the data from all studies. Study outcomes were summarized by PJT, EVC, and MM. They wrote the initial draft of the manuscript, and JB, JL, JMO, HR, and IB made significant revisions and contributions. All authors read and approved the final manuscript.

\section{Acknowledgements}

This work is part of the SPOTLIGHT project and is funded by the Seventh Framework Program (CORDIS FP7) of the European Commission, HEALTH (FP7-HEALTH-2011-two-stage), Grant agreement no. 278186. The content of this article reflects only the authors' views, and the European Commission is not liable for any use that may be made of the information contained therein.

\section{Other information}

This systematic review is reported in accordance with the PRISMA statement for reporting systematic reviews [21].

\section{Author details}

${ }^{1}$ Interdisciplinary Centre for the Study of Human Performance (CIPER), Faculty of Human Kinetics, University of Lisbon, Lisbon, Portugal. 'European Centre on Health of Societies in Transition, London School of Hygiene and Tropical Medicine, London, UK. ${ }^{3}$ Department of Nutrition Pitié-Salpetrière (AP-HP), Université Pierre et Marie Curie-Paris 6, Paris, France. ${ }^{4}$ UREN (Nutritional Epidemiology Research Unit), Université Paris 13, Sorbonne Paris Cité, Inserm (U557), Inra (U1125), Cnam, F-93017, Bobigny, France. ${ }^{5}$ Department of Movement and Sport Sciences, Ghent University, Ghent, Belgium.

${ }^{6}$ Department of General Practice and Elderly Care, The EMGO Institute for Health and Care Research, VU University Medical Center, Amsterdam, the Netherlands. ${ }^{7}$ Department of Epidemiology and Biostatistics, The EMGO Institute for Health and Care Research, VU University Medical Center, Amsterdam, the Netherlands.

Received: 12 November 2014 Accepted: 11 March 2015

Published online: 16 April 2015

\section{References}

1. Sniehotta FF, Scholz U, Schwarzer R. Bridging the intention-behaviour gap: planning, self-efficacy, and action control in the adoption and maintenance of physical exercise. Psychol Health. 2005;20:143-60.

2. Teixeira PJ, Going SB, Sardinha LB, Lohman TG. A review of psychosocial pre-treatment predictors of weight control. Obes Rev. 2005;6:43-65.

3. Maes S, Karoly P. Self-regulation assessment and intervention in physical health and illness: a review. Appl Psychol Int Rev. 2005;54:267-99.

4. Bandura A. The primacy of self-regulation in health promotion. Appl Psychol Health Well Being. 2005;54:245-54.

5. Ajzen I. From intentions to actions: a theory of planned behavior. In: Kuhl J, Beckmann J, editors. Action control. Berlin Heidelberg: Springer; 1985. p. 11-39.

6. Deci EL, Ryan RM. Intrinsic motivation and self-determination in human behavior. New York: Plenum; 1985. p. 113-48.

7. Heckhausen $\mathrm{H}$, Gollwitzer P. Thought contents and cognitive functioning in motivational versus volitional states of mind. Motiv Emot. 1987;11:101-20.

8. Schwarzer R. Modeling health behavior change: how to predict and modify the adoption and maintenance of health behaviors. Appl Psychol Health Well Being. 2008;57:1-29.

9. Carver CS, Scheier MF. On the self-regulation of behavior. New York: University Press; 1998.

10. Jensen MD, Ryan DH, Apovian CM, Ard JD, Comuzzie AG, Donato KA, et al. 2013 AHA/ACC/TOS guideline for the management of overweight and obesity in adults: a report of the American College of Cardiology/American Heart Association Task Force on Practice Guidelines and The Obesity Society. Circulation. 2014;129:5139-40.

11. Ackermann RT, Finch EA, Brizendine E, Zhou H, Marrero DG. Translating the diabetes prevention program into the community. The DEPLOY Pilot Study Am J Prev Med. 2008;35:357-63.

12. Eriksson MK, Franks PW, Eliasson M. A 3-year randomized trial of lifestyle intervention for cardiovascular risk reduction in the primary care setting: the Swedish Björknäs study. PLoS One. 2009;4:e5195.

13. Kinmonth AL, Wareham NJ, Hardeman W, Sutton S, Prevost AT, Fanshawe T, et al. Efficacy of a theory-based behavioural intervention to increase physical activity in an at-risk group in primary care (ProActive UK): a randomised trial. Lancet. 2008;371:41-8. 
14. van Sluijs EMF, van Poppel MNM, van Mechelen W. Stage-based lifestyle interventions in primary care: are they effective? Am J Prev Med. 2004:26:330-43.

15. Lakerveld J, Bot SD, Chinapaw MJ, van Tulder MW, Kostense PJ, Dekker JM, et al. Motivational interviewing and problem solving treatment to reduce type 2 diabetes and cardiovascular disease risk in real life: a randomized controlled trial. Int J Behav Nutr Phys Act. 2013;10:47.

16. Wing RR, Hill JO. Successful weight loss maintenance. Annu Rev Nutr. 2001;21:323-41.

17. Rossner S, Hammarstrand M, Hemmingsson E, Neovius M, Johansson K. Long-term weight loss and weight-loss maintenance strategies. Obes Rev. 2008;9:624-30.

18. Mata J, Silva MN, Vieira PN, Carraça EV, Andrade AM, Coutinho SR, et al. Motivational "spill-over" during weight control: increased self-determination and exercise intrinsic motivation predict eating self-regulation. Health Psychol. 2009;28:709-16.

19. Lakerveld J, Brug J, Bot S, Teixeira P, Rutter H, Woodward E, et al. Sustainable prevention of obesity through integrated strategies: the SPOTLIGHT project's conceptual framework and design. BMC Public Health. 2012;12:1471-2458.

20. Kraemer HC, Wilson GT, Fairburn CG, Agras WS. Mediators and moderators of treatment effects in randomized clinical trials. Arch Gen Psychiatry. 2002;59:877-83.

21. Liberati A, Altman DG, Tetzlaff J, Mulrow C, Gøtzsche PC, loannidis JP, et al. The PRISMA statement for reporting systematic reviews and meta-analyses of studies that evaluate health care interventions: explanation and elaboration. PLoS Med. 2009:6:e1000100

22. Elfhag K, Rossner S. Who succeeds in maintaining weight loss? A conceptual review of factors associated with weight loss maintenance and weight regain Obes Rev. 2005;6:67-85.

23. Friedman MA, Brownell KD. Psychological correlates of obesity: moving to the next research generation. Psychol Bull. 1995;117:3-20.

24. Barte J, ter Bogt NC, Bogers RP, Teixeira PJ, Blissmer B, Mori TA, et al. Maintenance of weight loss after lifestyle interventions for overweight and obesity, a systematic review. Obes Rev. 2010;11:899-906.

25. Franz MJ, Van Wormer JJ, Crain AL, Boucher JL, Histon T, Caplan W et al. Weight loss outcomes: a systematic review and meta-analysis of weight loss clinical trials with a minimum 1-year follow-up. J Am Diet Assoc. 2007;107:1755-67.

26. Curioni CC, Lourenco PM. Long-term weight loss after diet and exercise: a systematic review. Int J Obes (Lond). 2005;29:1168-74.

27. Higgins JP, Altman DG, Gøtzsche PC, Jüni $P$, Moher D, Oxman AD, et al. The Cochrane Collaboration's tool for assessing risk of bias in randomised trials. Br Med J. 2011;343:d5928.

28. Baron RM, Kenny DA. The moderator-mediator variable distinction in social psychological research: conceptual, strategic, and statistical considerations. J Pers Soc Psychol. 1986;51:1173-82.

29. MacKinnon DP, Fairchild AJ, Fritz MS. Mediation analysis. Annu Rev Psychol. 2007:58:593-614

30. Thomas BH, Ciliska D, Dobbins M, Micucci S. A process for systematically reviewing the literature: providing the research evidence for public health nursing interventions. Worldviews Evid Based Nurs. 2004;1:176-84.

31. Deeks JJ, Dinnes J, D'Amico R, Sowden AJ, Sakarovitch C, Song F, et al. Evaluating non-randomised intervention studies. Health Technol Assess. 2003; 7:iii-X.1-173.

32. Moher D, Cook DJ, Eastwood S, Olkin I, Rennie D, Stroup DF. Improving the quality of reports of meta-analyses of randomised controlled trials: the QUOROM statement. Lancet. 1999;354:1896-900.

33. Mackenbach JD, Rutter H, Compernolle S, Glonti K, Oppert JM, Charreire H, et al. Obesogenic environments: a systematic review of the association between the physical environment and adult weight status, the SPOTLIGHT project. BMC Public Health. 2014;14:233.

34. Lubans DR, Foster C, Biddle $S$. A review of mediators of behavior in interventions to promote physical activity among children and adolescents. Prev Med. 2008;47:463-70.

35. Rhodes RE, Pfaeffli LA. Mediators of physical activity behaviour change among adult non-clinical populations: a review update. Int J Behav Nutr Phys Act. 2010;7:37-48.

36. Silva MN, Markland D, Carraça EV, Vieira PN, Coutinho SR, Minderico CS, et al. Exercise autonomous motivation predicts 3-year weight loss in women. Med Sci Sports Exerc. 2011;43:728-37.
37. Annesi JJ, Unruh JL, Marti CN, Gorjala S, Tennant G. Effects of the coach approach intervention on adherence to exercise in obese women: assessing mediation of social cognitive theory factors. Res Q Exerc Sport. 2011:82:99-108.

38. Roesch SC, Norman GJ, Villodas F, Sallis JF, Patrick K. Intervention-mediated effects for adult physical activity: a latent growth curve analysis. Soc Sci Med. 2010;71:494-501.

39. Teixeira PJ, Silva MN, Coutinho SR, Palmeira AL, Mata J, Vieira PN, et al. Mediators of weight loss and weight loss maintenance in middle-aged women. Obesity (Silver Spring). 2010;18:725-35.

40. Perri MG, Limacher MC, Durning PE, Janicke DM, Lutes LD, Bobroff LB, et al. Extended-care programs for weight management in rural communities: the treatment of obesity in underserved rural settings (TOURS) randomized trial. Arch Intern Med. 2008;168:2347-54.

41. Burke V, Beilin LB, Cutt HE, Mansour J, Mori TA. Moderators and mediators of behaviour change in a lifestyle program for treated hypertensives: a randomized controlled trial (ADAPT). Health Educ Res. 2008;23:583-91.

42. Silva MN, Markland DM, Vieira PN, Coutinho SR, Carraça EV, Palmeira AL, et al. Helping overweight women become more active: need support and motivational regulations for different forms of physical activity. Psychol Sports Exerc. 2010;11:591-601.

43. Anderson-Bill ES, Winett RA, Wojcik JR, Williams DM. Aging and the social cognitive determinants of physical activity behavior and behavior change: evidence from the Guide to Health trial. J Aging Res. 2011. doi:10.4061/ 2011/505928.

44. Palmeira AL, Markland DA, Silva MN, Branco TL, Martins SC, Minderico CS, et al. Reciprocal effects among changes in weight, body image, and other psychological factors during behavioral obesity treatment: a mediation analysis. Int J Behav Nutr Phys Act. 2009;6:9.

45. Coughlin JW, Gullion CM, Brantley PJ, Stevens VJ, Bauck A, Champagne CM, et al. Behavioral mediators of treatment effects in the weight loss maintenance trial. Ann Behav Med. 2013;46:369-81.

46. Lee RE, O'Connor DP, Smith-Ray R, Mama SK, Medina AV, Reese-Smith JY, et al. Mediating effects of group cohesion on physical activity and diet in women of color: health is power. Am J Health Promot. 2012;26:e116-25.

47. Annesi JJ, Vaughn LL. Relationship of exercise volume with change in depression and its association with self-efficacy to control emotional eating in severely obese women. Adv Prev Med. 2011. doi:104061/2011/514271.

48. Annesi JJ, Gorjala S. Relationship of exercise program participation with weight loss in adults with severe obesity: assessing psychologically based mediators. South Med J. 2010;103:1119-23.

49. Linde JA, Rothman AJ, Baldwin AS, Jeffery RW. The impact of self-efficacy on behavior change and weight change among overweight participants in a weight loss trial. Health Psychol. 2006;25:282-91.

50. Riebe D, Blissmer B, Greene G, Caldwell M, Ruggiero L, Stillwell KM, et al. Long-term maintenance of exercise and healthy eating behaviors in overweight adults. Prev Med. 2005;40:769-78.

51. Gallagher Kl, Jakicic JM, Napolitano MA, Marcus BH. Psychosocial factors related to physical activity and weight loss in overweight women. Med Sci Sports Exerc. 2006;38:971-80.

52. Annesi JJ, Marti CN. Path analysis of exercise treatment-induced changes in psychological factors leading to weight loss. Psychol Health. 2011;26:1081-98.

53. Annesi JJ. Self-regulatory skills usage strengthens the relations of self-efficacy for improved eating, exercise, and weight in the severely obese: toward an explanatory model. Behav Med. 2011;37:71-6.

54. Annesi JJ. Behaviorally supported exercise predicts weight loss in obese adults through improvements in mood, self-efficacy, and self-regulation, rather than by caloric expenditure. Pers J. 2011;15:23-7.

55. Palmeira AL, Branco TL, Martins SC, Minderico CS, Silva MN, Vieira PN, et al. Change in body image and psychological well-being during behavioral obesity treatment: Associations with weight loss and maintenance. Body Image. 2010;7:187-93.

56. Teixeira PJ, Going SB, Houtkooper LB, Cussler EC, Metcalfe LL, Blew RM, et al Exercise motivation, eating, and body image variables as predictors of weight control. Med Sci Sports Exerc. 2006;38:179-88.

57. Annesi JJ. Relations of changes in physical self-appraisal and perceived energy with weight change in obese women beginning a supported exercise and nutrition information program. Soc Behav Personal Int J. 2007;35:295-300.

58. Moore SD, King AC, Kiernan M, Gardner CD. Outcome expectations and realizations as predictors of weight regain among dieters. Eat Behav. 2011;12:60-3. 
59. Haapala I, Barengo NC, Biggs S, Surakka L, Manninen P. Weight loss by mobile phone: a 1 year effectiveness study. Public Health Nutr. 2009;12:2382-91.

60. Annesi JJ, Whitaker AC. Weight loss and psychologic gain in obese women participants in a supported exercise intervention. The Permanente Journal. 2008;12:36-45.

61. Annesi JJ. Supported exercise improves controlled eating and weight through its effects on psychosocial factors: extending a systematic research program toward treatment development. Pers J. 2012;16:7-18.

62. Karhunen L, Lyly M, Lapveteläinen A, Kolehmainen M, Laaksonen DE, Lähteenmäki L, Poutanen K. Psychobehavioural factors are more strongly associated with successful weight management than predetermined satiety effect or other characteristics of diet. J Obes. 2012. doi:10.1155/2012/274068.

63. Wing RR, Papandonatos G, Fava JL, Gorin AA, Phelan S, McCaffery J, et al. Maintaining large weight losses: the role of behavioral and psychological factors. J Consult Clin Psychol. 2008;76:1015-21.

64. McGuire MT, Jeffery RW, French SA, Hannan PJ. The relationship between restraint and weight and weight-related behaviors among individuals in a community weight gain prevention trial. Int J Obes Relat Metab Disord. 2001:25:574-80.

65. Burke V, Mansour J, Mori TA, Beilin LB, Cutt HE, Wilson A. Changes in cognitive measures associated with a lifestyle program for treated hypertensives: a randomized controlled trial (ADAPT). Health Educ Res. 2008:23:202-17.

66. Warziski MT, Sereika SM, Styn MA, Music E, Burke LE. Changes in self-efficacy and dietary adherence: the impact on weight loss in the PREFER study. J Behav Med. 2008;31:81-92.

67. Annesi JJ, Mareno N. Temporal aspects of psychosocial predictors of increased fruit and vegetable intake in adults with severe obesity: mediation by physical activity. J Community Health. 2014;39:454-63.

68. Annesi J. Effects of treatment differences on psychosocial predictors of exercise and improved eating in obese, middle-age adults. J Phys Act Health. 2013;10:1024-31.

69. Annesi JJ, Porter KJ. Reciprocal effects of treatment-induced increases in exercise and improved eating, and their psychosocial correlates, in obese adults seeking weight loss: a field-based trial. Int J Behav Nutr Phys Act. 2013;10:133.

70. Fitzpatrick SL, Bandeen-Roche K, Stevens VJ, Coughlin JW, Rubin RR, Brantley PJ, et al. Examining the behavioral processes through which lifestyle interventions promote weight loss: results from the PREMIER Trial. Obesity (Silver Spring). 2014;22:1002-7.

71. Blair SN, Haskell WL, Ho P, Paffenbager Jr RS, Vranizan KM, Farquhar JW, et al. Assessment of habitual physical activity by a seven-day recall in a community survey and controlled experiments. Am J Epidemiol. 1985;122:794-804.

72. Godin G, Shephard RJ. Godin leisure-time exercise questionnaire. Med Sci Sports Exerc. 1997;29:S36-8.

73. Hayes AF. Introduction to mediation, moderation, and conditional process analysis: a regression-based approach. NY: Guilford Press; 2013.

74. Hagger MS. Theoretical integration in health psychology: unifying ideas and complementary explanations. Br J Health Psychol. 2009;14:189-94.

75. Michie S, Ashford S, Sniehotta FF, Dombrowski SU, Bishop A, French DP. A refined taxonomy of behaviour change techniques to help people change their physical activity and healthy eating behaviours: the CALO-RE taxonomy. Psychol Health. 2011;26:1479-98.

76. Cash TF. Cognitive-behavioral perspectives on body image. In: Cash TF, Pruzinsky T, editors. Body image: a handbook of theory, research, and clinical practice. New York: Guilford Press; 2002. p. 38-46.

77. Cash TF. A 'negative body image'. In: Cash TF, Pruzinsky T, editors. Body image: a handbook of theory, research, and clinical practice. New York: Guilford Press; 2002. p. 269-76.

78. Avalos L, Tylka TL, Wood-Barcalow N. The body appreciation scale: development and psychometric evaluation. Body Image. 2005;2:285-97.

79. Cash TF, Melnyk SE, Hrabosky Jl. The assessment of body image investment: an extensive revision of the appearance schemas inventory. Int J Eat Disord. 2004;35:305-16.

80. Verstuyf J, Patrick H, Vansteenkiste M, Teixeira PJ. Motivational dynamics of eating regulation: a self-determination theory perspective. Int J Behav Nutr Phys Act. 2012. 9:21. doi:10.1186/1479-5868-9-21.

81. Pelletier LG, Dion SC. An examination of general and specific motivational mechanisms for the relations between body dissatisfaction and eating behaviors. J Soc Clin Psychol. 2007;26:303-33.
82. Boone L, Soenens B, Braet C. Perfectionism, body dissatisfaction, and bulimic symptoms: the intervening role of perceived pressure to be thin and thin ideal internalization. J Soc Clin Psychol. 2011;30:1043-68.

83. Fabricatore A, Wadden T. Psychological functioning of obese individuals. Diabetes Spectrum. 2003;16:245-52

84. Schwartz MB, Brownell KD. Obesity and body image. Body Image. 2004;1:43-56.

85. Verstuyf J, Vansteenkiste M, Soenens B. Eating regulation and bulimic symptoms: the differential correlates of health-focused and appearancefocused eating regulation. Body Image. 2012;9:108-17.

86. Thogersen-Ntoumani C, Ntoumanis N, Nikitaras N. Unhealthy weight control behaviours in adolescent girls: a process model based on self-determination theory. Psychol Health. 2010;25:535-50.

87. Pelletier LG, Dion SC, D'Angelo MS, Reid RD. Why do you regulate what you eat? Relationships between forms of regulation, eating behaviors, sustained dietary behavior change, and psychological adjustment. Motiv Emot. 2004;28:245-77.

88. Ryan RM, Deci EL. Self-regulation and the problem of human autonomy: does psychology need choice, self-determination, and will? J Pers. 2006;74:1557-85

89. Deci EL, Ryan RM. The 'what' and 'why' of goal pursuits: human needs and the self-determination of behavior. Psychoanal Inq. 2000;11:227-68.

90. Ng JYY, Ntoumanis N, Thøgersen-Ntoumani C, Deci EL, Ryan RM, Duda JL, et al. Self-determination theory applied to health contexts: a meta-analysis. Perspect Psychol Sci. 2012;7:325-40.

91. Teixeira PJ, Carraça EV, Markland D, Silva MN, Ryan RM. Exercise, physical activity, and self-determination theory: a systematic review. Int J Behav Nutr Phys Act. 2012;9:78.

92. Armitage CJ, Conner M. Social cognition models and health behaviour: a structured review. Psychol Health. 2000;15:173-89.

93. Williams SL, French DP. What are the most effective intervention techniques for changing physical activity self-efficacy and physical activity behaviour and are they the same? Health Educ Res. 2011;26:308-22.

94. Bandura A. Self-efficacy: the exercise of control. New York: W.H. Freeman and Company; 1997

95. Westenhoefer J. Dietary restraint and disinhibition: is restraint a homogeneous construct? Appetite. 1991;16:45-55.

96. Bryant EJ, King NA, Blundell JE. Disinhibition: its effects on appetite and weight regulation. Obes Rev. 2008;9:409-19.

97. Kashdan TB, Rottenberg J. Psychological flexibility as a fundamental aspect of health. Clin Psychol Rev. 2010;30:865-78.

98. McCracken LM. Committed action: an application of the psychological flexibility model to activity patterns in chronic pain. J Pain. 2013;14:828-35.

99. Hayes SC, Luoma JB, Bond FW, Masuda A, Lillis J. Acceptance and commitment therapy: model, processes and outcomes. Behav Res Ther. 2006:44:1-25

100. Michie S, Abraham C, Whittington C, McAteer J, Gupta S. Effective techniques in healthy eating and physical activity interventions: a meta-regression. Health Psychol. 2009;28:690-701.

101. Hagger MS, Luszczynska A. Implementation intention and action planning interventions in health contexts: state of the research and proposals for the way forward. Appl Psychol Health Well Being. 2014;6:1-47.

102. Sniehotta FF. Towards a theory of intentional behaviour change: plans, planning, and self-regulation. Br J Health Psychol. 2009;14:261-73.

103. Hagger $M$, Chatzisarantis N. An integrated behavior change model for physical activity. Exerc Sport Sci Rev. 2014;42:62-9.

104. D'Angelo ME, Pelletier LG, Reid RD, Huta $V$. The roles of self-efficacy and motivation in the prediction of short- and long-term adherence to exercise among patients with coronary heart disease. Health Psychol. 2014;33:1344-53.

105. Carraça EV, Silva MN, Markland D, Vieira PN, Minderico CS, Sardinha LB, et al. Body image change and improved eating self-regulation in a weight management intervention in women. Int J Behav Nutr Phys Act. 2011;8:75-85.

106. Leong SL, Madden C, Gray A, Horwath C. Self-determined, autonomous regulation of eating behavior is related to lower body mass index in a nationwide survey of middle-aged women. J Acad Nutr Diet. 2012;112:1337-46.

107. Michie S, Richardson M, Johnston M, Abraham C, Francis J, Hardeman W, et al. The behavior change technique taxonomy ( $v 1$ ) of 93 hierarchically clustered techniques: building an international consensus for the reporting of behavior change interventions. Ann Behav Med. 2013;46:81-95.

108. Olander EK, Fletcher H, Williams S, Lou A, Turner A, French DP. What are the most effective techniques in changing obese individuals' physical activity 
self-efficacy and behaviour: a systematic review and meta-analysis. Int J Behav Nutr Phys Act. 2013;10:29.

109. Su Y-L, Reeve J. A meta-analysis of the effectiveness of intervention programs designed to support autonomy. Educ Psychol Rev. 2011;23:159-88.

110. Janssen V, Gucht V, Exel H, Maes S. A self-regulation lifestyle program for post-cardiac rehabilitation patients has long-term effects on exercise adherence. J Behav Med. 2014;37:308-21.

111. Miller W, Rollnick S. Motivational interviewing: helping people change. 3rd ed. NY: The Guilford Press; 2012.

112. Moyers TB, Martin T, Manuel JK, Hendrickson SM, Miller WR. Assessing competence in the use of motivational interviewing. J Subst Abuse Treat. 2005;28:19-26.

\section{Submit your next manuscript to BioMed Central} and take full advantage of:

- Convenient online submission

- Thorough peer review

- No space constraints or color figure charges

- Immediate publication on acceptance

- Inclusion in PubMed, CAS, Scopus and Google Scholar

- Research which is freely available for redistribution 